\title{
Identification of nuclear effects in neutrino and antineutrino interactions on nuclei using generalized final-state correlations
}

\author{
Xianguo $\mathrm{Lu}^{1, *}$ and Jan T. Sobczyk ${ }^{2, \dagger}$ \\ ${ }^{1}$ Department of Physics, University of Oxford, OX1 3PU Oxford, United Kingdom \\ ${ }^{2}$ Institute of Theoretical Physics, University of Wroctaw, pl. M. Borna 9, 50-204 Wroctaw, Poland
}

(Received 18 January 2019; published 21 May 2019)

\begin{abstract}
In the study of neutrino and antineutrino interactions in the $\mathrm{GeV}$ regime, kinematic imbalances of the final-state particles have sensitivities to different nuclear effects. Previous ideas based on neutrino quasielastic interactions [Lu, et al., Phys. Rev. C 94, 015503 (2016); Furmanski and Sobczyk, Phys. Rev. C 95, 065501 (2017)] are now generalized to antineutrino quasielastic interactions, as well as neutrino and antineutrino pion productions. Measurements of these generalized final-state correlations could provide unique and direct constraints on the nuclear response inherently different for neutrinos and antineutrinos and, therefore, delineate effects that could mimic charge-parity violation in neutrino oscillations.
\end{abstract}

DOI: 10.1103/PhysRevC.99.055504

\section{INTRODUCTION}

It has been well established that good understanding of (anti)neutrino-nucleus cross sections in the $\mathrm{GeV}$ regime is necessary for constraining systematic errors in long-baseline oscillation experiments $[1,2]$. The most challenging part of this effort is to describe nuclear effects. In the impulse approximation (IA) picture, which has a solid foundation in the few-GeV neutrino energy region, nuclear effects such as the target nucleon initial state (Fermi momentum and binding energy) and final-state interactions (FSIs) impact how individual scattering is seen in experimental setups. In particular, it is very important to understand well any imprint of nuclear effects on neutrino and antineutrino scattering that could be misunderstood and taken in the data analysis as a manifestation of charge-parity (CP) violation.

In addition to nuclear effects, the few-GeV energy region is complicated because of the overlap of many reaction channels, physics mechanisms, etc. A significant unknown is the contribution from the two-body current [called here also twoparticle-two-hole (2p2h)], a subject of many experimental and theoretical studies [3-12].

In the available models, events coming from the twobody current mechanism contribute almost entirely to the charged-current (CC) $0 \pi$ category, defined as having one charged lepton and no pion in the final state. They come there together with CC quasielastic (QE) events and also with pion production (RES) followed by absorption inside the nucleus.

\footnotetext{
*Xianguo.Lu@physics.ox.ac.uk

†jsobczyk@ift.uni.wroc.pl
}

Published by the American Physical Society under the terms of the Creative Commons Attribution 4.0 International license. Further distribution of this work must maintain attribution to the author(s) and the published article's title, journal citation, and DOI. Funded by $S C O A P^{3}$.
Measurements of muon momentum in $\mathrm{CC} 0 \pi$ events are very important for experiments like $\mathrm{T} 2 \mathrm{~K}$, where most of the information about the oscillation signal comes from detection of the final-state muons only. However, those measurements are not sufficient to put constraints on the amount of two-body current contributions. It is why there is a growing interest in measurements involving final-state protons. Interpretation of such measurements is challenging as it requires a good control of proton FSIs in Monte Carlo (MC) event generators [13]. This will become most exigent in liquid argon (LAr) experiments with a low-momentum proton detection threshold [14]. It is known that modeling the low-momentum nucleon in-medium cross section is most uncertain [15]. Challenges come together with opportunities and one may hope to learn from proton studies something new about nuclear physics.

Another challenge arising with the era of proton observables is that of the amount of information or organization of the data. One option is to measure and discuss multidimensional cross sections for muon and proton momenta [16]. Another possibility is to look at certain projections defined in such a way that their interpretation is simpler, pointing to particular details of physics mechanisms that are involved.

An intuitive way to look at proton observables is through single-transverse kinematic imbalances (single-TKIs) [17]. What is analyzed are only transverse projections of muon and proton momentum vectors on the plane perpendicular to the neutrino direction (known with a good precision). If CCQE interaction occurred on a nucleon at rest and if FSI effects were absent, the sum of the muon and proton momentum projections would vanish. Thus any deviation from zero tells us about nucleon Fermi motion, FSI effects, and also about other interaction mechanisms.

Recently two measurements of single-TKIs were performed by T2K [16] and MINERvA [18] experiments. Finalstate particle correlations are nontrivial in the presence of nuclear effects: the characteristic imprints from Fermi motion and intranuclear momentum transfer (IMT) (that in our 
nomenclature includes the impact of FSI and $2 \mathrm{p} 2 \mathrm{~h}$ dynamics) are readily identified in the measured cross sections. The new variable, transverse boosting angle $\delta \alpha_{\mathrm{T}}$ (for details, see Ref. [17]), preserves most of the Fermi motion isotropy and measures the strength of IMT. In the region $\delta \alpha_{\mathrm{T}}<90^{\circ}$, both T2K and MINERvA measurements are consistent, showing a common Fermi motion baseline; in the region $\delta \alpha_{\mathrm{T}}>90^{\circ}$ they differ strongly as $\delta \alpha_{\mathrm{T}}$ increases-an intriguing feature pointing to the energy dependence of IMT. In the MINERvA measurement, $\delta \alpha_{\mathrm{T}}$ also separates model predictions with boosting and dragging effects ("acceleration" vs "deceleration") of the FSI $[17,18]$, and therefore is able to isolate the peculiar elastic component of the GENIEhA FSI model [19].

A refinement of the kinematic imbalance studies was proposed in Ref. [20]. The basic observation was that in the IA regime the assumption that the interaction mechanism was CCQE and no FSI occurred allows to resolve the kinematics completely once the final-state muon and proton are measured. The additional piece of information used here comes from the longitudinal components of the final-state muon and proton momenta. The new proposed observable is emulated nucleon momentum $p_{\mathrm{N}}$. Its first measurement was done by the MINERvA Collaboration [18]. In the data there are three interesting regions in $p_{\mathrm{N}}$. A pronounced peak at $p_{\mathrm{N}} \sim 200 \mathrm{MeV} / c$ is, according to MC simulations, dominated overwhelmingly by CCQE events without FSI. Then there is a tail region $p_{\mathrm{N}} \gtrsim 400 \mathrm{MeV} / c$ and the intermediate region in between with a lot of structures allowing for detailed studies of interaction mechanisms and FSI effects. The CCQE peak in $p_{\mathrm{N}}$ shows the neutron momentum distribution and it may seem surprising that a neutrino measurement with all its limitations allows for a nice visualization of the basic nucleon featurethat of Fermi motion.

Keeping in mind the usefulness of experimental studies of nuclear target reactions with the single-TKI and emulated nucleon momentum observables, we would like to extend this approach to other experimental situations. Several important reactions are discussed in the same theoretical framework. We argue that when put together they provide a powerful source of information about reaction mechanisms and nuclear effects.

Processes to be discussed are the following (the $N$ below stands for "at least one"):

$$
\begin{aligned}
& \nu 0 \pi N \mathrm{p}: \nu \mathrm{A} \rightarrow \ell^{-} \mathrm{pX}, \\
& \bar{\nu} 0 \pi N \mathrm{p}: \bar{\nu} \mathrm{A} \rightarrow \ell^{+} \mathrm{pX}, \\
& \nu 1 \pi N \mathrm{p}: \nu \mathrm{A} \rightarrow \ell^{-} \mathrm{p} \pi^{+} \mathrm{X}, \\
& \bar{\nu} 1 \pi N \mathrm{p}: \bar{\nu} \mathrm{A} \rightarrow \ell^{+} \mathrm{p} \pi^{-} \mathrm{X},
\end{aligned}
$$

where $\mathrm{X}$ is a final-state hadronic system consisting of the nuclear remnant with possible additional knocked-out nucleons but without mesons. It is assumed that one charged lepton, at least one proton, and for Eqs. (3) and (4) additionally one charged pion, are detected. These include major $v / \bar{v}$ interaction channels at current and future accelerator-based neutrino experiments. The first investigation of the $1 \pi$ channels [Eqs. (3) and (4)] using single-TKI and double-TKI [21] was presented in Ref. [22].
The goal of this paper is to propose experimental probes for surgical diagnostics in nuclear effects in both neutrino and antineutrino $\mathrm{CC}$ interactions. The potential of the new observables is illustrated by performing numerical simulations using GiBUU and NuWro generators with the MINERvA and $\mathrm{T} 2 \mathrm{~K}$ beam fluxes. The plan of the paper is as follows. In Secs. II-IV, we present the signal definitions, the formulas of the generalized final-state correlations, and the simulation details. In Secs. V and VI, we discuss the model predictions and the implications.

\section{UNDERLYING INTERACTION DYNAMICS}

The underlying interaction dynamics of Eqs. (1)-(4) in IA and neglecting FSI are summarized as follows (to simplify the discussion, we neglect in this section but not in the numerical computations diffractive and higher resonant pion production):

$$
\begin{aligned}
& v \mathrm{n} \rightarrow \ell^{-} \mathrm{p}, \\
& \text { not applicable, } \\
& v \mathrm{p} \rightarrow \ell^{-} \Delta^{++} \rightarrow \ell^{-} \mathrm{p} \pi^{+}, \\
& \bar{v} \mathrm{p} \rightarrow \ell^{+} \Delta^{0} \rightarrow \ell^{+} \mathrm{p} \pi^{-} .
\end{aligned}
$$

The process in Eq. (2) is forbidden in IA without FSI due to charge imbalance.

In 2 p2h dynamics neglecting FSI, additional reaction channels underlying Eqs. (1) and (2) are

$$
\begin{gathered}
\nu \mathrm{n} \mathrm{N} \rightarrow \ell^{-} \mathrm{p} \mathrm{N}, \\
\bar{v} \mathrm{p} \mathrm{p} \rightarrow \ell^{+} \mathrm{n} \mathrm{p},
\end{gathered}
$$

where the above $\mathrm{N}$ stands for either a proton or a neutron. One can see that Eq. (2) becomes possible as a result of the $2 \mathrm{p} 2 \mathrm{~h}$ process. We disregard pion production in the $2 \mathrm{p} 2 \mathrm{~h}$ mechanism, about which very little is known.

When FSI sets in, many new scenarios contributing to reactions in Eqs (1)-(4) become possible. Most importantly, the pions resulting from primary interactions can be absorbed, and the nucleons can knock out other nucleons or even pions seen in the final state. Among other channels, Eq. (2) is proposed here for its pure nuclear-effect origin. Its unique feature, as shown in following sections, is the strongly reduced influence from the Fermi motion.

\section{GENERALIZED FINAL-STATE CORRELATIONS}

The nuclear target processes in Eqs. (1)-(4) can be summarized as

$$
v / \bar{v}+\mathrm{A} \rightarrow \ell+\widetilde{\mathrm{N}}+\mathrm{X},
$$

where $\widetilde{N}$ is a proton in Eqs. (1) and (2) and a p $\pi$ pair in Eqs. (3) and (4). Similarly, in IA the reactions in Eqs. (5), (7), and (8) can be summarized as

$$
v / \bar{v}+\mathrm{N} \rightarrow \ell+\tilde{\mathrm{N}} .
$$

Accordingly, the definitions of single-TKI given in Ref. [17] are generalized so that

$$
\delta \vec{p}_{\mathrm{T}}=\vec{p}_{\mathrm{T}}^{\ell}+\vec{p}_{\mathrm{T}}^{\widetilde{\mathrm{N}}}
$$


where $\vec{p}_{\mathrm{T}}^{\ell}$ and $\vec{p}_{\mathrm{T}}^{\widetilde{N}}$ are the transverse momenta of the lepton and $\widetilde{\mathrm{N}}$, respectively. The definition of the transverse boosting angle keeps its original form:

$$
\delta \alpha_{\mathrm{T}} \equiv \arccos \frac{-\vec{p}_{\mathrm{T}}^{\ell} \cdot \delta \vec{p}_{\mathrm{T}}}{p_{\mathrm{T}}^{\ell} \delta p_{\mathrm{T}}} .
$$

Assuming that the target nucleus was at rest and no other particles were knocked out (i.e., $\mathrm{X}$ is the nuclear remnant of mass $M_{\mathrm{X}}$ ), one can resolve the kinematics of the process following the steps from Ref. [20]. The result for the longitudinal component of the target nucleon momentum is

$$
\begin{aligned}
p_{L}= & \frac{1}{2}\left(M_{\mathrm{A}}+k_{\mathrm{L}}^{\ell}+p_{\mathrm{L}}^{\widetilde{\mathrm{N}}}-E_{\ell}-E_{\widetilde{\mathrm{N}}}\right) \\
& -\frac{\delta p_{\mathrm{T}}^{2}+M_{\mathrm{X}}^{2}}{2\left(M_{\mathrm{A}}+k_{\mathrm{L}}^{\ell}+p_{\mathrm{L}}^{\widetilde{\mathrm{N}}}-E_{\ell}-E_{\widetilde{\mathrm{N}}}\right)},
\end{aligned}
$$

where $M_{\mathrm{A}}$ is the target nucleus mass, and $k_{\mathrm{L}}^{\ell}\left(p_{\mathrm{L}}^{\widetilde{\mathrm{N}}}\right)$ and $E_{\ell}$ $\left(E_{\widetilde{\mathrm{N}}}\right)$ are the longitudinal momentum and energy of $\ell(\widetilde{\mathrm{N}})$, respectively.

The emulated nucleon momentum is defined as

$$
p_{\mathrm{N}} \equiv \sqrt{\delta \vec{p}_{\mathrm{T}}^{2}+p_{L}^{2}} .
$$

The value of $M_{\mathrm{X}}$ can be expressed in terms of the target nucleus mass $M_{\mathrm{A}}$ and the proton/neutron mean excitation energies $\langle\epsilon\rangle_{\mathrm{p} / \mathrm{n}}$ :

$$
M_{\mathrm{X}}=M_{\mathrm{A}}-M_{\mathrm{p} / \mathrm{n}}+\langle\epsilon\rangle_{\mathrm{p} / \mathrm{n}},
$$

where $M_{\mathrm{p} / \mathrm{n}}$ is proton or neutron mass. The values used in this paper are $\langle\epsilon\rangle_{\mathrm{n}}=28.7 \mathrm{MeV}$ and $\langle\epsilon\rangle_{\mathrm{p}}=26.1 \mathrm{MeV}$ (see Table 8 of Ref. [23]).

\section{SIMULATIONS}

\section{A. NuWro}

NuWro [24] is a versatile MC neutrino event generator developed over last 13 years at Wrocław University. It provides a complete description of neutrino and antineutrino interactions on arbitrary nucleon and nuclear targets in the energy range from $\sim 100 \mathrm{MeV}$ to $\sim 1 \mathrm{TeV}$. The basic interaction modes on a free-nucleon target are CCQE [see Eq. (5), and its neutral current counterpart]; RES, which covers a region of invariant hadronic mass $W \leqslant 1.6 \mathrm{GeV}$ [the dominant RES process is $\Delta(1232)$-resonance excitation as in Eqs. (7) and (8)]; and DIS (jargon in the neutrino MC community for shallow and deep inelastic scattering [13]) which includes all the inelastic processes with $W \geqslant 1.6 \mathrm{GeV}$.

In the case of neutrino-nucleus scattering, two new interaction modes are $\mathrm{COH}$, for coherent pion production, and $\mathrm{MEC}$, for two-body current processes, called also $2 \mathrm{p} 2 \mathrm{~h}$.

Neutrino-nucleus CCQE, RES, DIS, and MEC reactions are modeled as a two-step process; the primary interaction on one or two nucleons is followed by FSI.

NuWro FSI effects are described by a custom-made semiclassical intranuclear cascade (INC) model [24]. It includes pion absorption and charge-exchange reactions treated according to the model of Oset et al. [25,26]. Values of nucleon-nucleon in-medium cross section are based on the computations from Ref. [27].
In this paper we use NuWro configuration 17.09. CCQE is described with the local Fermi gas (LFG) model, and the standard vector and axial form factors with the axial mass value of $1.03 \mathrm{GeV}$. RPA effects are added following Ref. [28]. RES is based on $\mathrm{N}-\Delta(1232)$ transition axial form factors found in Ref. [29] by a fit to ANL and BNL pion production data. The nonresonant contribution is added incoherently as explained in Ref. [30]. The nuclear target pion production cross section is reduced due to in-medium self-energy implemented in the approximate way using results of Ref. [31]. Finite $\Delta(1232)$ life-time effects are also included [24], as well as realistic angular distributions of pions resulting from $\Delta(1232)$ decays [32]. DIS is based on inclusive neutrino cross-section computations of Bodek and Yang with hadronization modeled using PYTHIA fragmentation routines. MEC is based on the Nieves et al. model [5] with a momentum transfer cut $q \leqslant 1.2 \mathrm{GeV} / c$ [6]. As for the MEC hadronic part a model from Ref. [33] is used. It is assumed that in $85 \%$ of MEC events the interaction occurs on a proton-neutron pair $[34,35]$.

\section{B. GiBUU}

GiBUU [36,37] is a theoretical model and also an event generator describing nuclear interactions with nuclei, including photon-, lepton-, hadron-, and nucleus-nucleus reactions, with a consistent treatment of nuclear effects and a sophisticated kinetic hadronic transport framework. In these calculations, both the initial- and final-state hadrons are embedded in a coordinate- and momentum-dependent potential. The 2017 version is used in this study.

The target-nucleon momentum is sampled like in the LFG approach, but due to the nuclear potential the bound nucleon has an effective mass. In this approach, inclusion of RPA correlations are not needed (see Refs. [38-40]). The axial mass parameter in the dipole form factor for the quasielastic scattering is set to $1 \mathrm{GeV}$.

In the pion production kinematic region $(W<2 \mathrm{GeV})$, the vector couplings and transition form factors are determined by the MAID analysis [41]. The axial part for heavier resonances is determined by partially conserved axial current (PCAC) arguments and an assumption of a dipole form factor with the axial mass parameter of $1 \mathrm{GeV}$, whereas for $\Delta(1232)$ the axial part was obtained by a fit to bubble-chamber data $[42,43]$. The nonresonant contributions (together with the interference one) are added in an incoherent way. Free spectral functions without in-medium corrections are used for the $\Delta$ resonance [44]. GiBUU does not provide predictions for the coherent pion production. Inelastic processes at $W$ above $2 \mathrm{GeV}$ are described as DIS by PYTHIA [45].

The $2 \mathrm{p} 2 \mathrm{~h}$ contribution in GiBUU is fully determined by the structure functions $W_{1}$ and $W_{3}$. By neglecting the longitudinal part of the response, both structure functions are directly related to the structure functions measured in electron-nucleus scattering [46]. The relative numbers of initial neutron-proton, neutron-neutron, and proton-proton pairs are determined by combinatorics arguments.

After primary interactions, final-state particles are transported on-shell in phase-space volumes where quantum statistical effects like Pauli blocking are handled. GiBUU 
allows for an off-shell transport of hadrons but the results do not change much, and therefore this option is not used in this study. Pion absorption in FSIs is modeled as two- and three-nucleon processes [44]; charge-exchange reactions are described in Ref. [47].

\section{MINERvA selection criteria}

Predictions are calculated with the NuMI low-energy beam flux [48] on carbon targets with the following particle selection:

(1) Muon: $\theta_{\mu}<20^{\circ}, 1.5<p_{\mu}<10 \mathrm{GeV} / c$.

(2) Proton: $\theta_{\mathrm{p}}<70^{\circ}, 0.45<p_{\mathrm{p}}<1.2 \mathrm{GeV} / c$, and at least one proton satisfies the above criteria and the most energetic one is selected in the analysis.

(3) Charged pion: $\theta_{\pi}<70^{\circ}, 75<T_{\pi}<400 \mathrm{MeV}$, and exactly one charged pion satisfies the above criteria.

(4) No mesons otherwise.

Here $p, T$, and $\theta$ are the particle momentum, kinetic energy, and angle with respect to the neutrino direction. These selection criteria are derived from MINERvA measurements (for example, Refs. [18,49]). In nonmagnetized detectors like the MINERvA scintillator tracker, particle momentum is determined by range. The $T$ upper cuts for protons and pions are to remove particles undergoing secondary interactions in the detector, to guarantee a precise momentum measurement.

\section{T2K selection criteria}

Predictions are calculated with the T2K beam flux [50] on carbon targets with the following particle selection:

(1) Muon: $\theta_{\mu}<126.87^{\circ}\left(\cos \theta_{\mu}>-0.6\right), \quad p_{\mu}>0.25$ $\mathrm{GeV} / c$.

(2) Proton: $\theta_{\mathrm{p}}<66.42^{\circ}\left(\cos \theta_{\mathrm{p}}>0.4\right), 0.45<p_{\mathrm{p}}<1$ $\mathrm{GeV} / c$, and at least one proton satisfies the above criteria and the most energetic one is selected in the analysis.

(3) Charged pion: $\theta_{\pi}<70^{\circ}, 75<T_{\pi}<400 \mathrm{MeV}$, and exactly one pion satisfies the above criteria.

(4) No mesons otherwise.

These selection criteria are derived from $\mathrm{T} 2 \mathrm{~K}$ measurements (for example, Refs. [16,51]). In T2K, the time projection chamber (TPC) of the near detector can provide precise momentum measurements of pions above $T_{\pi}=400 \mathrm{MeV}$. The upper $T_{\pi}$ cut here is to have a consistent signal definition as in MINERvA so that the predictions can be compared within similar phase space.

\section{RESULTS}

In Fig. 1, results for the $\nu 0 \pi N$ p selection are shown. The top two panels present the results with the MINERvA flux as predicted by GiBUU and NuWro. The bottom two panels show the NuWro results with both MINERvA and T2K fluxes. The theoretical predictions contain contributions from several dynamical mechanisms: QE, RES+DIS with pion absorption,
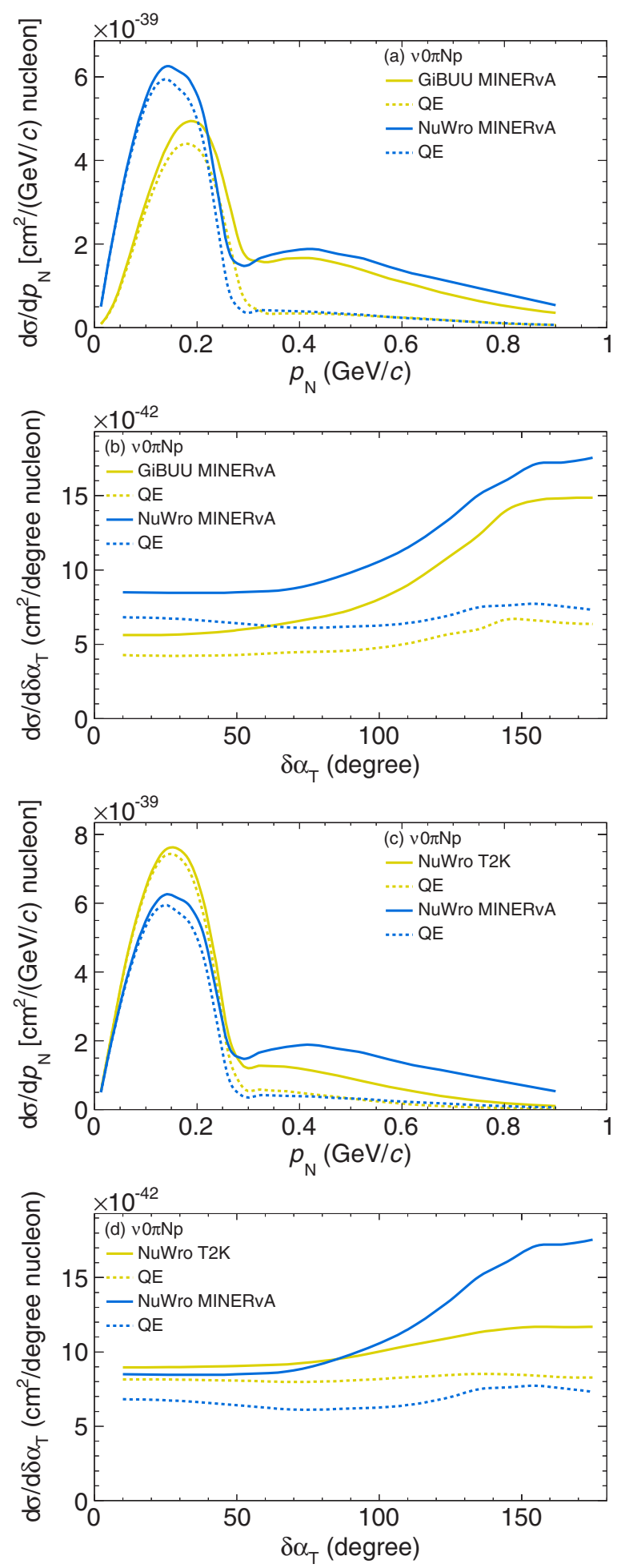

FIG. 1. GiBUU and NuWro predictions for the $\nu 0 \pi N \mathrm{p}$ selection. Differential cross sections in $p_{\mathrm{N}}$ and $\delta \alpha_{\mathrm{T}}$, respectively, are compared between the two generators (upper two panels) and between MINERvA and T2K using NuWro (lower two panels). The corresponding $\mathrm{QE}$ components are shown.

and $2 \mathrm{p} 2 \mathrm{~h}$. We show RES+DIS rather than RES and DIS separately because the RES and DIS definitions in NuWro and GiBUU do not match but the sums do. 
As discussed in Ref. [20], $p_{\mathrm{N}}$ is defined in such a way that, for QE events where the knocked-out proton does not suffer from FSI effects, it is equal to the target-neutron momentum. This explains the peak in the $p_{\mathrm{N}}$ distribution at 150-200 $\mathrm{MeV} / c$ : it comes from the neutron Fermi motion. The comparison between NuWro and GiBUU indicates that the initial state is modeled differently. It is clear that with the experimental data it is possible to discriminate between theoretical models (see Refs. [18,52]). For example, the hole spectral function approach [53] as implemented in NuWro provides much better agreement with the data than LFG with RPA corrections (see Ref. [18]). For both experiments the shape and position of the peak in the $p_{\mathrm{N}}$ distribution predicted by NuWro are very similar and the difference is mostly in its height (the T2K peak is higher). Our understanding is that MINERvA has on average more energetic protons which are removed from the Fermi motion peak by stronger FSI to the right tail.

For $\delta \alpha_{\mathrm{T}}$, the nonflatness of the distribution of the QE events indicates the strength of the FSI experienced by the knockedout protons. In the bottom panel we see that the fraction of non-QE events gradually increases towards the large $\delta \alpha_{\mathrm{T}}$ direction, and at $\delta \alpha_{\mathrm{T}}=180^{\circ}$ the beam-energy dependence becomes maximal.

In Fig. 2 results for the $\bar{\nu} 0 \pi N p$ selection are shown in the same format as in Fig. 1. This channel only includes QE events with charge-exchange nucleon FSI; therefore, compared to the $\nu 0 \pi N \mathrm{p}$ channel the dominant Fermi motion peak is absent, and the rise of $\delta \alpha_{\mathrm{T}}$ is much steeper. An interesting observation with this selection is that the GiBUU and NuWro overall predictions are very similar in shape and normalization, and yet this agreement turns out to be accidental since individual contributions from interaction modes are quite different. This is illustrated with the $2 \mathrm{p} 2 \mathrm{~h}$ contributions shown separately.

$\nu 0 \pi N \mathrm{p}$ and $\bar{\nu} 0 \pi N \mathrm{p}$ contain complementary information about FSI and $2 \mathrm{p} 2 \mathrm{~h}$ mechanisms. For the $\nu 0 \pi N \mathrm{p}$ selection, QE FSI events are those with quasielastic proton rescattering. In the $\bar{\nu} 0 \pi N p$ selection, nucleon charge-exchange FSI is needed for the QE mechanism; the 2p2h contribution comes either from proton-proton initial pairs without FSI or from proton-neutron pairs with charge-exchange FSI. NuWro assumes a much bigger fraction of initial proton-neutron pairs than GiBUU. We see that the two channels are sensitive to different details of the nucleon FSI and 2p2h mechanisms, and therefore a combined analysis of both channels would help to reveal the full picture of these dynamics.

The channels $\nu 1 \pi N p$ and $\bar{\nu} 1 \pi N p$ (Figs. 3 and 4) are dominated by RES+DIS contributions. The variable $p_{\mathrm{N}}$ is defined in such a way that, for events with $\Delta(1232)$ excitation decaying into a charged pion and a proton but not suffering from FSI effects, it is equal to the initial-nucleon momentum. This time the target nucleon is a proton and the difference between $\nu 1 \pi N \mathrm{p}$ and $\bar{v} 1 \pi N \mathrm{p}$ is in the charge of the final-state pion. In both cases, a clear Fermi motion peak is predicted by GiBUU and NuWro, but again with different shapes. This peak provides the most direct constraint on the Fermi motion of the initial-state proton, which has not been yet directly studied in neutrino interactions. The overall amount of RES+DIS events without FSI is similar in both models, as demonstrated
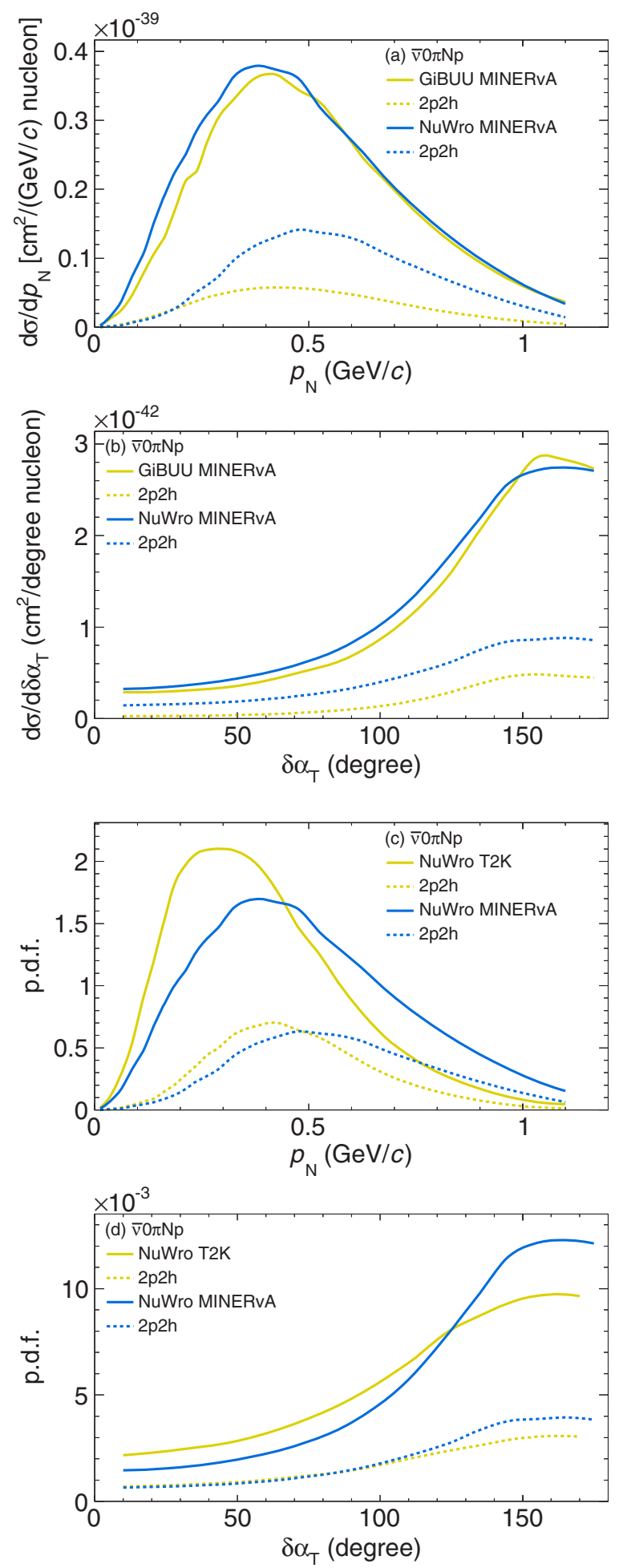

FIG. 2. Model comparisons for $\bar{\nu} 0 \pi N p$ in the same layout as in Fig. 1. The comparisons between MINERvA and T2K in the lower two panels are shown in shape. The $2 \mathrm{p} 2 \mathrm{~h}$ components are shown.

by the cross section at $\delta \alpha_{\mathrm{T}} \rightarrow 0$. And yet, the $\delta \alpha_{\mathrm{T}}$ rising trend indicates the different FSI strength in the two models. Also, by comparing the rising trend of $\delta \alpha_{\mathrm{T}}$ between the two channels, one can conclude that in both models the $\bar{\nu} 1 \pi N \mathrm{p}$ channel suffers stronger FSI, making a higher tail in $p_{\mathrm{N}}$. 

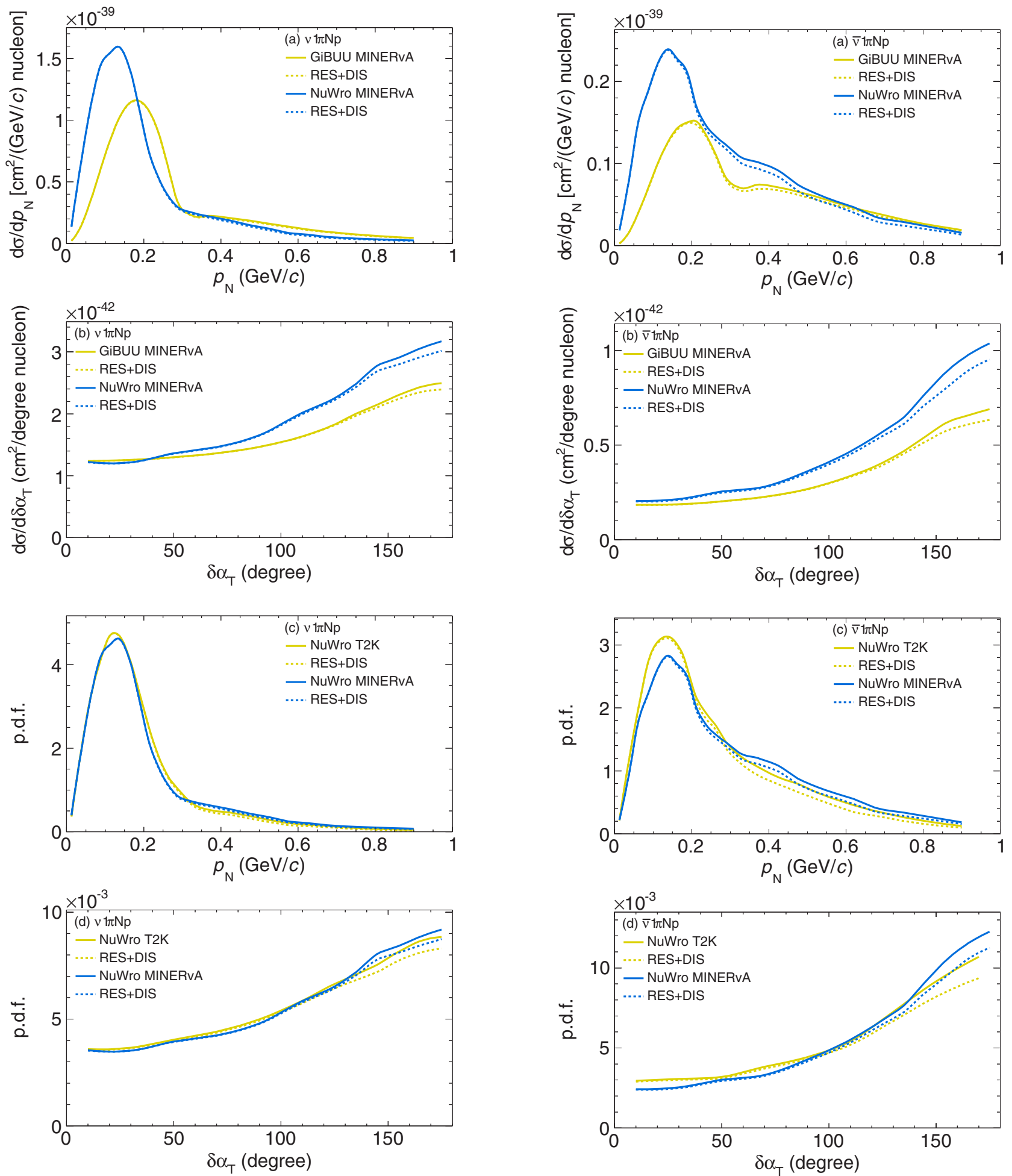

FIG. 3. Model comparisons for $\nu 1 \pi N p$ in the same layout as in Fig. 2. The RES+DIS components are shown.

The calculations show that, in the kinematic regions of $\nu 1 \pi N \mathrm{p}$ and $\bar{\nu} 1 \pi N \mathrm{p}$ probed by MINERvA and T2K experiments, the shape of $p_{\mathrm{N}}$ and $\delta \alpha_{\mathrm{T}}$ would depend only weakly on the neutrino energy. This is a very strong statement to be verified by experiments.

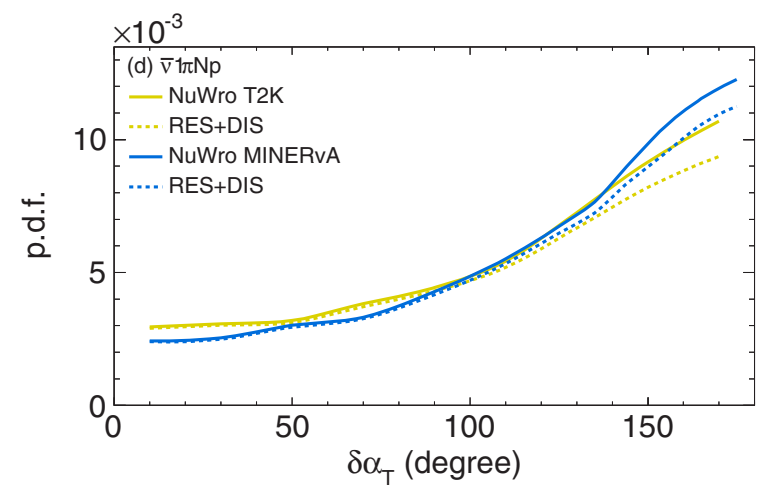

FIG. 4. Model comparisons for $\bar{\nu} 1 \pi N \mathrm{p}$ in the same layout as in Fig. 3.

\section{DISCUSSION AND OUTLOOK}

The next-generation long-baseline neutrino oscillation experiments DUNE and Hyper-Kamiokande aim to measure $\mathrm{CP}$ violation based on a comparison of the neutrino and antineutrino oscillation patterns. To achieve this goal a very 
good control of nuclear effects in (anti)neutrino scattering is necessary. In particular, it is very important to control nuclear effects that are inherently distinct for neutrino and antineutrino scattering. The proposed generalized final-state correlations among the charged lepton and hadrons are minimally affected by nucleon-level phenomena and the beam flux [17]. They directly reveal details of the nuclear effects and allow to test theoretical models.

In water Cherenkov detectors one looks mainly at CCQE events and it is critical to analyze the oscillation signal with a model describing precisely the distributions of the chargedlepton kinetic energy and angle. Recent studies [1,2,23] have shown the effects of the initial states in measuring the oscillation parameters. In the $\delta_{\mathrm{CP}}$ measurements where chargedlepton kinematics are used to infer the neutrino energy, understanding of the underlying neutron and proton Fermi motion is particularly important. Because the observed Fermi motion is weighted by the underlying (anti)neutrino-nucleon cross section, a direct measurement of proton Fermi motion in the $\nu 1 \pi N \mathrm{p}$ and $\bar{\nu} 1 \pi N \mathrm{p}$ channels using $p_{\mathrm{N}}$ could provide valuable knowledge of the response of the constituent proton to the different electroweak probes mediated by $W^{ \pm}$bosons, which could mimic $\mathrm{CP}$ violation in neutrino oscillations.

It would be interesting to use the proposed observables in LAr experiments. Argon is a heavier nuclear target which makes nucleon FSI effects stronger. However, in LAr detectors lower-momentum knocked-out protons with weaker FSI effects are also reconstructed. In the recent measurement of electron scattering on an argon target [54], the Fermi motion peaks from carbon and argon are shown to have different shapes. While Fermi motion of the constituent nucleons in argon nuclei can be inferred with electron scattering, it can be determined in situ in neutrino interactions by measuring $p_{\mathrm{N}}$ in the $\nu 0 \pi N \mathrm{p}, \nu 1 \pi N \mathrm{p}$, and $\bar{\nu} 1 \pi N \mathrm{p}$ channels, the response to the axial current elicited.

As is suggested by the MINERvA $\nu 0 \pi N p$ measurement [18], state-of-the-art generators fail in the transition region of $p_{\mathrm{N}}$ between the Fermi motion peak and the region that is dominated by FSI and $2 \mathrm{p} 2 \mathrm{~h}$. Without the Fermi motion peak in the $\bar{v} 0 \pi N \mathrm{p}$ channel, the source of this model deficit could be determined. More importantly, as Ref. [18] suggests that this intermediate $p_{\mathrm{N}}$ region is where the MINERvA empirical $2 \mathrm{p} 2 \mathrm{~h}$ enhancement $[55,56]$ is strongest, one might suspect that the deficit could be related to the modeling of $2 \mathrm{p} 2 \mathrm{~h}$. In the $\bar{\nu} 0 \pi N \mathrm{p}$ channel, the ambiguity caused by the Fermi motion tail in constraining $2 \mathrm{p} 2 \mathrm{~h}$ models is removed, potentially allowing a better understanding of this complicated mechanism. As such, the interplay of several dynamical mechanisms - the initial state, QE, RES, and 2p2h interactions, and nucleon and pion FSIs-could be resolved.
It is important to note that, for determination of the initial state, neutral-pion production can also be considered:

$$
v \mathrm{~A} \rightarrow \ell^{-} \mathrm{p} \pi^{0} \mathrm{X}
$$

with a nucleon-level interaction,

$$
v \mathrm{n} \rightarrow \ell^{-} \mathrm{p} \pi^{0}
$$

The generalized final-state correlations from Eq. (18) have similar sensitivity to Fermi motion as from the channel in Eq. (1). In this paper we focused on the channels with charged pions in the final state, but a measurement in this channel could provide complementary information.

Apart from nuclear-effect measurements, by selecting the Fermi motion peak in the $p_{\mathrm{N}}$ distribution one can select a high-purity sample of genuine QE and RES events that do not experience FSIs in the $0 \pi$ [Eq. (1)] and $1 \pi$ [Eqs. (3)-(4)] channels, respectively. In such samples the neutrino energy can be precisely reconstructed, as was first illustrated for the QE events in Ref. [20].

The generalized final-state correlations focus on kinematics imbalances in exclusive reactions, which can be complemented by calorimetric inclusive measurements around the vertex region [55,56]. For example, a better $p_{\mathrm{N}}$ peak measurement could be achieved by imposing a cut on the vertex energy, so that events other than no-FSI QE/RES are removed from the $0 \pi / 1 \pi$ channel(s).

Finally, the selection of three charged particles required in the $v 1 \pi N \mathrm{p}$ and $\bar{\nu} 1 \pi N \mathrm{p}$ channels has important experimental implications. As was first proposed and discussed in Refs. [21,57], it enables to extract, on an event-by-event basis, neutrino- and antineutrino-hydrogen interactions from compound targets that contain hydrogen atoms. In addition to the double-TKI [21], the single-TKI (imbalance between the charged lepton and hadrons) and $p_{\mathrm{N}}$ in principle could also provide separation power between interactions on hydrogen and heavier nuclei when the detector responses are optimized.

\section{ACKNOWLEDGMENTS}

We thank Trung Le for the discussions about pion secondary interactions in MINERvA, and Ulrich Mosel for helpful comments on the manuscript. J.T.S. was supported by NCN Opus Grant No. 2016/21/B/ST2/01092, and also by the Polish Ministry of Science and Higher Education, Grant No. DIR/WK/2017/05. X.L. was supported by the UK Science and Technology Facilities Council. X.L. thanks Kevin McFarland and Ulrich Mosel for valuable discussions.
[1] L. Alvarez-Ruso et al., Prog. Part. Nucl. Phys. 100, 1 (2018).

[2] M. Betancourt et al., Phys. Rep. 773-774, 1 (2018).

[3] J. Marteau, Eur. Phys. J. A 5, 183 (1999).

[4] M. Martini, M. Ericson, G. Chanfray, and J. Marteau, Phys. Rev. C 81, 045502 (2010).

[5] J. Nieves, I. R. Simo, and M. J. Vicente Vacas, Phys. Rev. C 83, 045501 (2011).
[6] R. Gran, J. Nieves, F. Sanchez, and M. J. Vicente Vacas, Phys. Rev. D 88, 113007 (2013).

[7] R. González-Jiménez, G. D. Megias, M. B. Barbaro, J. A. Caballero, and T. W. Donnelly, Phys. Rev. C 90, 035501 (2014).

[8] T. Van Cuyck, N. Jachowicz, R. González-Jiménez, M. Martini, V. Pandey, J. Ryckebusch, and N. Van Dessel, Phys. Rev. C 94, 024611 (2016). 
[9] T. Van Cuyck, N. Jachowicz, R. González-Jiménez, J. Ryckebusch, and N. Van Dessel, Phys. Rev. C 95, 054611 (2017).

[10] O. Benhar, A. Lovato, and N. Rocco, Phys. Rev. C 92, 024602 (2015).

[11] A. Lovato, S. Gandolfi, J. Carlson, E. Lusk, S. C. Pieper, and R. Schiavilla, Phys. Rev. C 97, 022502(R) (2018).

[12] N. Rocco, C. Barbieri, O. Benhar, A. De Pace, and A. Lovato, Phys. Rev. C 99, 025502 (2019).

[13] H. Gallagher and Y. Hayato ( Particle Data Group), Chin. Phys. C 38, 090001 (2014).

[14] R. Acciarri et al., Phys. Rev. D 90, 012008 (2014).

[15] G.-Q. Li and R. Machleidt, Phys. Rev. C 48, 1702 (1993).

[16] K. Abe et al. (The T2K Collaboration), Phys. Rev. D 98, 032003 (2018).

[17] X.-G. Lu, L. Pickering, S. Dolan, G. Barr, D. Coplowe, Y. Uchida, D. Wark, M. O. Wascko, A. Weber, and T. Yuan, Phys. Rev. C 94, 015503 (2016).

[18] X.-G. Lu et al. (MINERvA), Phys. Rev. Lett. 121, 022504 (2018).

[19] C. Andreopoulos, C. Barry, S. Dytman, H. Gallagher, T. Golan, R. Hatcher, G. Perdue, and J. Yarba, arXiv:1510.05494.

[20] A. P. Furmanski and J. T. Sobczyk, Phys. Rev. C 95, 065501 (2017).

[21] X.-G. Lu, D. Coplowe, R. Shah, G. Barr, D. Wark, and A. Weber, Phys. Rev. D 92, 051302(R) (2015).

[22] L. Pickering and X.-G. Lu, arXiv:1606.04403.

[23] A. Bodek and T. Cai, Eur. Phys. J. C 79, 293 (2019).

[24] T. Golan, C. Juszczak, and J. T. Sobczyk, Phys. Rev. C 86, 015505 (2012).

[25] L. L. Salcedo, E. Oset, M. J. Vicente-Vacas, and C. GarciaRecio, Nucl. Phys. A 484, 557 (1988).

[26] E. Oset and L. L. Salcedo, Nucl. Phys. A 468, 631 (1987).

[27] V. R. Pandharipande and S. C. Pieper, Phys. Rev. C 45, 791 (1992).

[28] K. M. Graczyk and J. T. Sobczyk, Eur. Phys. J. C 31, 177 (2003).

[29] K. M. Graczyk, D. Kielczewska, P. Przewlocki, and J. T. Sobczyk, Phys. Rev. D 80, 093001 (2009).

[30] C. Juszczak, J. A. Nowak, and J. T. Sobczyk, Nucl. Phys. Proc. Suppl. 159, 211 (2006).

[31] J. T. Sobczyk and J. Żmuda, Phys. Rev. C 87, 065503 (2013).

[32] J. T. Sobczyk and J. Zmuda, Phys. Rev. C 91, 045501 (2015).
[33] J. T. Sobczyk, Phys. Rev. C 86, 015504 (2012).

[34] J. Carlson, J. Jourdan, R. Schiavilla, and I. Sick, Phys. Rev. C 65, 024002 (2002).

[35] R. Subedi et al., Science 320, 1476 (2008).

[36] O. Buss, T. Gaitanos, K. Gallmeister, H. van Hees, M. Kaskulov, O. Lalakulich, A. B. Larionov, T. Leitner, J. Weil, and U. Mosel, Phys. Rep. 512, 1 (2012).

[37] K. Gallmeister, U. Mosel, and J. Weil, Phys. Rev. C 94, 035502 (2016)

[38] M. Martini, N. Jachowicz, M. Ericson, V. Pandey, T. Van Cuyck, and N. Van Dessel, Phys. Rev. C 94, 015501 (2016).

[39] J. Nieves and J. E. Sobczyk, Ann. Phys. 383, 455 (2017).

[40] J. E. Sobczyk, Phys. Rev. C 96, 045501 (2017).

[41] D. Drechsel, S. S. Kamalov, and L. Tiator, Eur. Phys. J. A 34, 69 (2007).

[42] G. M. Radecky, V. E. Barnes, D. D. Carmony, A. F. Garfinkel, M. Derrick, E. Fernandez, L. Hyman, G. Levman et al., Phys. Rev. D 25, 1161 (1982); 26, 3297(E) (1982).

[43] O. Lalakulich, T. Leitner, O. Buss, and U. Mosel, Phys. Rev. D 82, 093001 (2010).

[44] U. Mosel and K. Gallmeister, Phys. Rev. C 97, 045501 (2018).

[45] T. Leitner, O. Buss, L. Alvarez-Ruso, and U. Mosel, Phys. Rev. C 79, 034601 (2009).

[46] P. E. Bosted and V. Mamyan, arXiv:1203.2262.

[47] O. Buss, L. Alvarez-Ruso, A. B. Larionov, and U. Mosel, Phys. Rev. C 74, 044610 (2006).

[48] L. Aliaga et al. (MINERvA Collaboration), Phys. Rev. D 94, 092005 (2016); 95, 039903 (2017).

[49] A. Mislivec et al. (MINERvA Collaboration), Phys. Rev. D 97, 032014 (2018).

[50] K. Abe et al. (T2K Collaboration), Nucl. Instrum. Methods Phys. Res. Sect. A 659, 106 (2011).

[51] K. Abe et al. (T2K Collaboration), Phys. Rev. D 95, 012010 (2017).

[52] S. Dolan, arXiv: 1810.06043.

[53] O. Benhar, A. Fabrocini, S. Fantoni, and I. Sick, Nucl. Phys. A 579, 493 (1994).

[54] H. Dai et al., arXiv:1810.10575.

[55] P. A. Rodrigues et al. (MINERvA Collaboration), Phys. Rev. Lett. 116, 071802 (2016); 121, 209902 (2018).

[56] R. Gran et al. (MINERvA Collaboration), Phys. Rev. Lett. 120, 221805 (2018).

[57] X.-G. Lu, JPS Conf. Proc. 12, 010034 (2016). 